\title{
Correction to: Severing telicity from result
}

\section{On two types of resultative compound verb in Dongying Mandarin}

\section{Chenchen Song ${ }^{1}$}

\section{Correction to: J East Asian Linguist https://doi.org/10.1007/s10831-018-9170-8}

In the online publication of above-mentioned article a few corrections were incorrectly carried out.

In Introduction, first paragraph below example (1) the correct sentence reads: Second, I will further adapt the split-VP model along the lines of Cuervo (2003) (event decomposition) and Travis (2010) (Inner Aspect).

In Sect. 7.1, second paragraph below example (52) the correct sentence reads: A solution to this problem is implied in Travis' (2010) three loci of telicity marking, i.e. V1, Asp, and X in (34), or Voice, Asp, and $v B E(-\sqrt{ })$ in (40).

In Sect. 7.2, paragraph above example (57) the correct text reads:

In addition, the bare liu passes the telic tests in Sect. 3 well. As in (57), it is incompatible with an additional telicizing liu in completive context, conditional bunegation, and the potential-liu construction.

\footnotetext{
The original article has been corrected.

The original article can be found online at https://doi.org/10.1007/s10831-018-9170-8.

Chenchen Song

cs791@cam.ac.uk

1 Theoretical and Applied Linguistics, Faculty of Modern and Medieval Languages, University of Cambridge, Sidgwick Avenue, Cambridge CB3 9DA, UK
} 